\title{
UNA EXPERIENCIA DE EVALUACIÓN FORMATIVA EN EL MÁSTER DE FORMACIÓN DEL PROFESORADO DE EDUCACIÓN SECUNDARIA
}

\author{
A Formative Assessment Experience in Master of Teacher Training of Secondary \\ School
}

Uma Experiência de Avaliação Formativa no Mestrado de Formação de Professores de Ensino Secundário

Cristina Vallés Rapp (1)

Cristina Gil Puente (2)

Ma Antonia López-Luengo (3)

(1) Universidad de Valladolid, España. Teléfono: +34921112235/2325 Correo electrónico: cvalles@dce.uva.es

(2) Universidad de Valladolid, España. Teléfono: +34921112235/2325 Correo electrónico: cgil@dce.uva.es

(3) Universidad de Valladolid, España. Teléfono: +34921112235/2325 Correo electrónico: mlopezl@dce.uva.es

\section{Resumen}

En la presente comunicación se muestra una experiencia de evaluación formativa en el marco del Máster de Formación del Profesorado de Secundaria, concretamente, en el desarrollo de una asignatura denominada "Innovación Docente". Los objetivos han sido diseñar el sistema de evaluación de la asignatura, implementarlo y valorarlo. Los datos se han obtenido a través de la observación participante, el análisis de documentos y los cuestionarios cumplimentados por los participantes a lo largo del curso. Los resultados muestran la implicación y satisfacción por parte de los estudiantes, así como un alto grado de coordinación del equipo docente implicado.

Palabras clave: Educación superior; formación inicial de profesorado; evaluación formativa; innovación educativa, educación secundaria

\begin{abstract}
This communication shows an experience of Formative Assessment of the Master of Teacher Training of Secondary School, in the subject "Teaching Innovation". The objective has been designed of the Assessment process teaching programme, implementation and evaluation. Data have been obtained with participant observation, document analysis and questionnaires to participants. The results show the involvement


and satisfaction of the students, as well as a great degree of coordination the teaching team involved.

Keywords: Higher education; initial teacher training; formative assessment; teaching innovation; secondary education

\section{Resumo}

A presente comunicação mostra uma experiência de avaliação formativa no âmbito do Mestrado de Formação de Professores do Secundário, nomeadamente, no desenvolvimento da disciplina "Inovação Docente". Os objetivos consistiram no desenho do sistema de avaliação da disciplina, sua implementação e avaliação. Os dados foram obtidos através da observação participante, análise de documentos e questionários preenchidos pelos participantes durante o curso. Os resultados mostram o envolvimento e a satisfação dos estudantes, bem como um alto grau de coordenação da equipa docenteenvolvida.

Palavras-chave: Educação superior; formação inicial de professores; avaliação formativa; inovação docente; ensino secundário

\section{Introducción}

Se presenta una experiencia en la que se trata la adquisición de competencias que establece la orden ECI/3858/2007 de 27 de diciembre en el marco del Máster de Formación del Profesorado de Secundaria, Especialidad en Tecnología Agraria, Alimentaria y Forestal, concretamente, en el desarrollo de una asignatura denominada "Innovación Docente" que se ha implementado por segunda vez por este grupo de profesoras.

El equipo docente pretende que las clases presenciales incluyan estrategias didácticas innovadoras como flipped learning (Sams \& Bergmann, 2014), rutinas de pensamiento (Ritchhart, Church, \& Morrison, 2011), o gamificación (Prieto, Díaz, Monserrat, \& Reyes, 2014) entre otras. Se trata de conseguir que el alumno tome conciencia sobre este hecho y contraste con otras experiencias formativas vividas en su historia académica. Además, se solicita al alumnado que diseñe secuencias innovadoras para el nivel en el que realizará su docencia. Es reseñable el hecho de que apenas existen 
experiencias publicadas de innovación docente sobre asignaturas en grados de Formación Profesional.

En este caso se plantea un sistema de evaluación global de la asignatura, un reto importante, pues está impartida por tres profesoras y ello supone un alto grado de coordinación para obtener buenos resultados.

\section{Contextualización}

El trabajo que se aborda se desarrolla en una asignatura cuatrimestral con una carga crediticia de 3 ECTS que se está implementando durante el curso académico 2018-19. En cuanto a los destinatarios se trata de un grupo de alumnos muy reducido ( 7 alumnos) que provienen de una formación muy técnica (son ingenieros) y la mayoría tiene experiencia profesional en su campo, pero no en docencia, sin embargo presentan gran capacidad de trabajo e interés por los temas que se abordan en la asignatura.

\section{Diseño y desarrollo}

Se ha llevado a cabo el diseño del programa docente de la asignatura, su implementación y evaluación a través de la observación participante, el análisis de documentos y los cuestionarios cumplimentados por los participantes a lo largo del curso.

En la experiencia presentada se implementa un sistema de evaluación formativa. Las actividades de aprendizaje consisten en: exposiciones participativas para presentar los contenidos fundamentales; lectura y análisis crítico de experiencias docentes innovadoras en Formación Profesional publicadas, intercambio de experiencias y reflexión sobre la práctica, así como tutorías de seguimiento del trabajo del alumnado (de forma presencial y on-line). Cobra especial relevancia la Secuencia de Enseñanza Aprendizaje (SEA) cuyo objetivo general es diseñar, poner en práctica una propuesta didáctica durante el Prácticum y evaluar los resultados obtenidos. En este encargo se pide a los estudiantes que tengan en cuenta la inclusión de las TIC como recurso educativo de forma justificada y atender, al menos, a tres de sus utilidades pedagógicas señaladas por Cañal (2016), entre otras su función evaluativa. Por otro lado, se requiere a los estudiantes que apliquen un sistema de evaluación formativa (López Pastor, 2012) y elaboren para ello, de forma justificada, los instrumentos necesarios. En todas las actividades de aprendizaje las profesoras implicadas facilitan feedback, bien de forma 
escrita, si la tarea se entrega a través de la plataforma de aprendizaje, bien de forma verbal, si la tarea se desarrolla en el aula.

\section{Evaluación}

Todos los alumnos han desarrollado la propuesta con gran calidad, entusiasmo e implicación en las aulas, en este sentido se han cubierto sobradamente las expectativas al inicio del proceso. Se han recogido testimonios de los alumnos que reflejan la utilidad de la experiencia y su satisfacción durante el trabajo realizado en las aulas, pero también la dificultad y la alta carga de trabajo que ha supuesto la elaboración y revisión de las tareas. A continuación, se presenta una de las afirmaciones de los estudiantes:

"Esta actividad ha supuesto un reto mayor que el resto de mi labor docente realizada durante el periodo de prácticas. En primer lugar, tenía una gran motivación por ver la respuesta del alumnado ante este método innovador de aprender (...)” (Sujeto 4).

Al tratarse de un número de alumnos tan reducido se consigue un seguimiento personal y cercano, y un mayor dinamismo en el aula. En las sesiones ha resultado muy fácil generar diálogo y situaciones de debate, la implicación y el interés mostrado por los alumnos ha facilitado la tarea docente y ha supuesto un estímulo importante para el profesorado implicado.

El alumnado ha podido observar cómo los contenidos se vinculan a través de actividades interconectadas en las que se emplean metodologías activas y evaluación formativa. El equipo de profesoras el curso pasado asumió de manera precipitada la docencia de la asignatura $\mathrm{y}$, aunque hubo coordinación, algunos estudiantes manifestaron falta de coherencia en la evaluación y calificación. Este hecho y el propio deseo de mejora de las docentes, así como el convencimiento de que el profesorado en formación necesita buenos ejemplos, impulsaron el desarrollo de esta experiencia que se ha conseguido gracias a un mayor tiempo en la planificación de la misma.

La metodología seguida en la asignatura resulta novedosa en el contexto de la formación inicial de Profesorado de Secundaria; así lo manifiesta el alumnado del máster en el cuestionario sobre la experiencia llevada a cabo durante el Prácticum y su evaluación. Este alumnado valora especialmente el hecho de que la asignatura esté centrada en aspectos prácticos y muestran su interés en reuniones de seguimiento del Título donde han demandado más asignaturas de este tipo y el aumento de créditos de la asignatura que nos ocupa.

Una experiencia de evaluación formativa en el Máster de Formación del Profesorado de Educación 
Consideramos que la práctica llevada a cabo es efectiva, demuestra un impacto positivo y tangible de mejora que se aprecia en la aplicación por parte de los alumnos de ciertos aprendizajes a su práctica en el aula de Formación Profesional. Además, es una práctica sostenible, porque el aprendizaje se consigue de forma progresiva y acompañada, no es inmediato pero se espera que tenga repercusión en la trayectoria docente de los futuros profesores. Se trata de una práctica fácilmente replicable, es posible utilizarla como modelo para desarrollarla incluso por los propios alumnos en las aulas de Educación Secundaria.

\section{Referencias}

Cañal, P. (Coord.) (2016). Didáctica de las Ciencias Experimentales en Educación Primaria. Madrid: Paraninfo.

López Pastor, V. M. (2012). Evaluación formativa y compartida en la universidad: clarificación de conceptos y propuestas de intervención desde la Red Interuniversitaria de Evaluación Formativa. Psychology, Society \& Education, 4 (1), 117-130

Orden ECI/3858/2007 de 27 de diciembre por la que se establecen los requisitos para la verificación de los títulos universitarios oficiales que habiliten para el ejercicio de las profesiones de Profesor de Educación Secundaria Obligatoria y Bachillerato, Formación Profesional y Enseñanzas de Idiomas. 53751 - 53752. Recuperado de: https:/www.boe.es/eli/es/o/2007/12/27/eci3858/con

Prieto A., Díaz D., Monserrat J., \& Reyes E. (2014). Experiencias de aplicación de estrategias de gamificación a entornos de aprendizaje universitario ReVisión, 7 (2), 76-92

Ritchhart, R., Church, M., \& Morrison, K. (2011). Making thinking visible. How to Promote Engagement, Undestanding, and Independence for All Learners. San Francisco: Jossey-Bass.

Sams, A., \& Bergmann, J. (2014). Flipped learning gateway to student engagement. Washington: International Society for Technology in Education. 